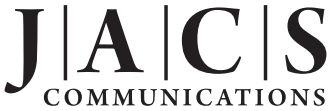

\section{Oxidative Template for Conducting Polymer Nanoclips}

\author{
Zhen Liu, ${ }^{\dagger}$ Xinyu Zhang, ${ }^{, \dagger}$ Selcuk Poyraz, ${ }^{\dagger}$ Sumedh P. Surwade, ${ }^{\ddagger}$ and Sanjeev K. Manohar, ${ }^{\star \star}$ \\ Department of Polymer and Fiber Engineering, Auburn University, Auburn, Alabama 36849, and Department of \\ Chemical Engineering, University of Massachusetts Lowell, Lowell Massachusetts 01854
}

Received July 6, 2010; E-mail: xzz0004@auburn.edu; sanjeev_manohar@uml.edu

\begin{abstract}
Bulk quantities of electronic conducting polymers such as polyaniline, polypyrrole, and poly(3,4-ethylenedioxythiophene), having an unusual 2D nanoclip-like morphology is described using a general oxidative template assembly route which is orchestrated by an insoluble complex formed between an anionic oxidant $\left(\mathrm{S}_{2} \mathrm{O}_{8}{ }^{2-}\right)$ and a cationic surfactant.
\end{abstract}

We describe a facile, one-step, general oxidative template assembly (OTA) approach to synthesize bulk quantities of electronic conducting polymers such as polyaniline (PANI), polypyrrole (PPy), and poly(3,4-ethylenedioxythiophene) (PEDOT), having an unusual nanoclip-like morphology. The morphology is orchestrated by an insoluble complex formed between an anionic oxidant $\left(\mathrm{S}_{2} \mathrm{O}_{8}{ }^{2-}\right)$ and a cationic surfactant. The as-produced conducting polymers possess two-dimensional (2-D) nanostructures instead of granular structures without the template. This OTA approach could be used to control the bulk morphology and permit large-scale production of conducting polymers or other precipitation polymerization systems, e.g., sol-gel technique for metal oxides.

Nanostructured conducting polymers are attractive due to their unique chemical, and physical properties and potential applications as building blocks of nanoelectronic devices. A variety of approaches are employed to synthesize nanostructured conducting polymers, including template-assisted, ${ }^{1-3}$ interfacial, ${ }^{4}$ and seeding polymerization. 5 However, most reports highlight strategies to synthesize 0-D or 1-D nanostructures (nanospheres, wires), and very few on higher order dimensional nanostructures, such as $2-\mathrm{D}^{7,8}$ or even 3-D nanostructures, which can be readily applied in the fabrication of electronic devices. ${ }^{9}$ This paper describes the synthesis of 2-D conducting polymer nanoclips using an oxidative template composed of cetrimonium cations and peroxydisulfate anions, $(\text { CTA })_{2} \mathrm{~S}_{2} \mathrm{O}_{8}$, that has potential for use as a "universal" template for all major classes of conducting polymers that are synthesized by precipitation polymerization.

In a typical synthesis of polypyrrole nanoclips, $0.01 \mathrm{M}$ cetrimonium bromide $\left(\left(\mathrm{C}_{16} \mathrm{H}_{33}\right) \mathrm{N}\left(\mathrm{CH}_{3}\right)_{3} \mathrm{Br}\right)(\mathrm{CTAB})$ is dispersed in 60 $\mathrm{mL}$ of $1 \mathrm{M}$ aqueous $\mathrm{HCl}$ in an ice bath. After magnetic stirring for $10 \mathrm{~min}, 0.03 \mathrm{M}\left(\mathrm{NH}_{4}\right)_{2} \mathrm{~S}_{2} \mathrm{O}_{8}$ (APS) is added, and stirring is continued for an additional $10 \mathrm{~min}$, resulting in a white precipitate of a CTAB/APS complex that functions as an oxidative template for polymerization. The reaction mixture is cooled to $0-3{ }^{\circ} \mathrm{C}$, and pyrrole $(0.12 \mathrm{M})$ monomer is added. The polymerization is allowed to proceed at $0-3{ }^{\circ} \mathrm{C}$ for $24 \mathrm{~h}$. A black precipitate of hydrochloridedoped polypyrrole, $\mathrm{PPy} \cdot \mathrm{Cl}$, forms that is suction-filtered and then washed with copious amounts of $1 \mathrm{M}$ aqueous $\mathrm{HCl}(3 \times 100 \mathrm{~mL})$ and acetone $(3 \times 100 \mathrm{~mL})$. The powder is freeze-dried for $12 \mathrm{~h}$, yielding $\sim 100 \mathrm{mg}$ of $\mathrm{PPy} \cdot \mathrm{Cl}$ nanoclips.

\footnotetext{
$\dagger$ Auburn University.

* University of Massachusetts Lowell.
}

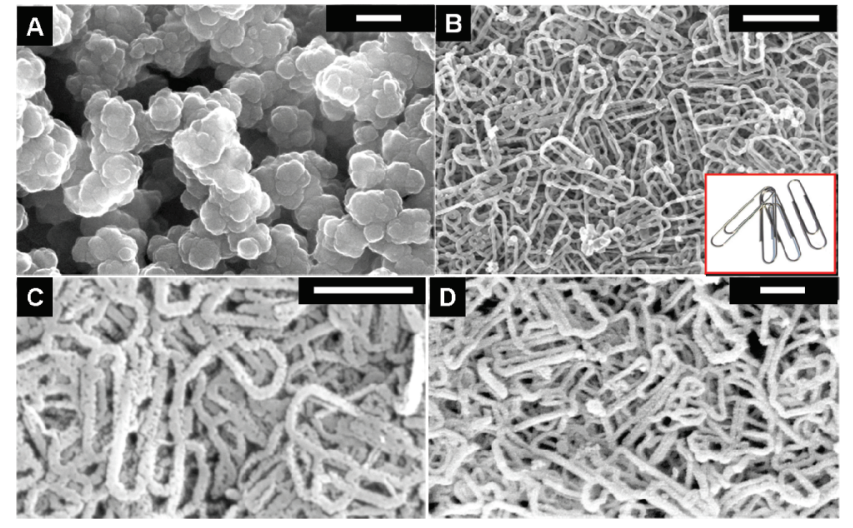

Figure 1. $\mathrm{SEM}$ images of (A) granular $\mathrm{PPy} \cdot \mathrm{Cl}($ scale bar, $200 \mathrm{~nm}),(\mathrm{B})$ $\mathrm{PPy} \cdot \mathrm{Cl}$ nanoclips (scale bar, $1 \mu \mathrm{m}$; inset, digital picture of paper clips), (C) $\mathrm{PANI} \cdot \mathrm{HCl}$ nanoclips (scale bar, $1 \mu \mathrm{m}$ ), and (D) PEDOT $\cdot \mathrm{Cl}$ nanoclips (scale bar, $1 \mu \mathrm{m}$ ).

A scanning electron microscopy (SEM) image of the PPy powder obtained above shows a homogeneous nanoclip morphology with average diameter in the $50-70 \mathrm{~nm}$ range (Figure $1 \mathrm{~B}$ ), which is to be contrasted with the granular morphology observed in the absence of CTAB (Figure 1A). The OTA method is general and can be used to synthesize other conducting polymers. For example, PANI and PEDOT are synthesized using a very similar procedure (see Supporting Information). In the case of PEDOT, a longer reaction time is needed, which is consistent with the larger monomer size and hydrophobic nature of EDOT that slows the diffusion into the CTAB/APS template complex.

Both PANI and PEDOT powder possess nanoclip morphology (Figure 1C,D), with diameters of $\sim 100 \mathrm{~nm}$ for PANI and $\sim 200$ $\mathrm{nm}$ for PEDOT. The difference in diameters could be explained by the varying sizes of the individual polymer chains and the packing patterns of different polymers. The nanoclips are analytically pure and highly conducting; e.g., the elemental analysis of PPy $\cdot \mathrm{Cl}$ nanoclips: $\mathrm{C}, 54.63 ; \mathrm{H}, 4.41 ; \mathrm{N}, 14.71 ; \mathrm{O}, 13.00 ; \mathrm{Cl}, 12.27$; $\mathrm{Br}$, 0.89; total, 99.91, is consistent with the chemical composition $(\mathrm{PPy})(\mathrm{CTAB})_{0.01}\left(\mathrm{Cl}^{-}\right)_{0.33}\left(\mathrm{H}_{2} \mathrm{O}\right)_{0.78}$. Although there is trace-level surfactant residue in the as-produced polymer, the doping level is $33 \%$, which is consistent with previously published studies. ${ }^{6}$

The four-probe pressed-pellet direct current conductivity values of these oxidatively p-doped conducting polymer nanoclips are in the range of 2-5 S/cm, similar to their conventional granular counterparts (no surfactants), although for polypyrrole it is 3 orders of magnitude higher than for previously reported polypyrrole nanoribbons synthesized using CTAB. ${ }^{10}$ Although spectroscopically the nanoclips are essentially identical to their corresponding granular counterparts (Figure S3, Supporting Information), there is a significant difference in their capacitance values. Using cyclic voltammetry, the specific capacitance value calculated by an established method ${ }^{11}$ was $\sim 190 \mathrm{~F} / \mathrm{g}$ for PPy $\cdot \mathrm{Cl}$ nanoclips, compared to $\sim 35 \mathrm{~F} / \mathrm{g}$ for granular $\mathrm{PPy} \cdot \mathrm{Cl}$ (Figure $\mathrm{S} 6$, 

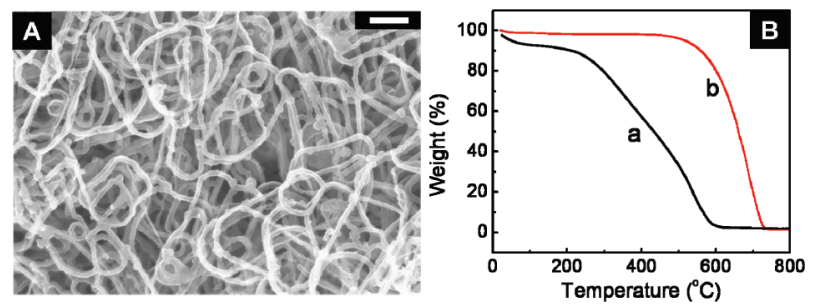

Figure 2. (A) $\mathrm{SEM}$ image of microwave-heated $\mathrm{PPy} \cdot \mathrm{Cl}($ scale bar, $300 \mathrm{~nm}$ ), (B) TGA of $\mathrm{PPy} \cdot \mathrm{Cl}$ nanoclips before (a) and after (b) microwave heating.

Supporting Information). We believe this could be important in nextgeneration energy storage devices.

These nanoclips can also be used as precursors for microwaveinitiated nanocarbonization. ${ }^{12}$ In a standardized procedure, $30 \mathrm{mg}$ of as-synthesized nanoclips is placed in a glass vial and heated in a conventional microwave oven, in ambient air, for 5-6 min. Continuous sparks appear on the polymer nanoclips surface owing to the strong absorption of microwave irradiation by conducting polymers. The vials become red-hot during the microwave heating, and the polymer loses heteroatoms, dopants, etc. from the polymer backbone to produce carbon powder that has retained its nanoclip morphology. Elemental analysis shows that the carbon content increases from $55 \%$ to $\sim 80 \%$ after microwave heating for $6 \mathrm{~min}$. Thermogravimetric analysis (TGA) data (Figure 2B) show a surprisingly large improvement in thermal stability after microwave heating; e.g., whereas $\mathrm{PPy} \cdot \mathrm{Cl}$ nanoclips decompose completely at $\sim 600{ }^{\circ} \mathrm{C}$, nanocarbon clips are very stable, with $<5 \%$ weight loss. Although microwave heating destroys some nanoclip features, e.g., loss of sharp edges, etc., a significant part of the nanostructured morphology is retained.

The exact mechanism of the micelle packing, leading to the final polymer assembly, remains unclear, although a possible rationale is proposed. Based on X-ray photoelectron spectroscopy of the reactive template, the surface composition of sulfur and nitrogen is $2.5 \%$ and $1.1 \%$, respectively, which represents a $1: 2$ ratio of $\mathrm{S}_{2} \mathrm{O}_{8}{ }^{2-}$ anions and cetrimonium cations, consistent with the chemical structure (CTA $)_{2} \mathrm{~S}_{2} \mathrm{O}_{8}$. We believe lamellar micelles are present that are composed of a folded, twin-tailed complex of (CTA) ${ }_{2} \mathrm{~S}_{2} \mathrm{O}_{8}$ in $1 \mathrm{M}$ aqueous $\mathrm{HCl}$ (Scheme 1) whose formation is favored by the high ionic strength of the reaction medium which reduces repulsion between the charged hydrophilic headgroups. Such a finding is different from the previously reported linear structure obtained in a magnetically stirred system in deionized water. ${ }^{2}$ A template structure is also discussed in a previous study employing a magnetically stirred system in DI water, ${ }^{7}$ which yielded different bulk morphologies and polymer having relatively low conductivity. In contrast, the aqueous $\mathrm{HCl}$ system used in our study: (i) promotes higher conductivity in the precipitated polymers, (ii) leads to different assembly mechanisms due to enhanced ionic strength, which orients the micelles in a specific arrangement, and (iii) increases the positive charge on the monomers via protonation. ${ }^{3}$ The positively charged monomers will preferably migrate to the interface between the micellar complex and aqueous $\mathrm{HCl}$ medium (Scheme 1), where the oxidation of the monomers will occur. Since excess $\mathrm{S}_{2} \mathrm{O}_{8}{ }^{2-}$ is used, the template will remain intact until all $\mathrm{S}_{2} \mathrm{O}_{8}{ }^{2-}$ in solution is consumed; i.e., the template will eventually be broken by consumption of the $\mathrm{S}_{2} \mathrm{O}_{8}{ }^{2-}$ ions, which bridge the cetrimonium ions. Although significant challenges remain in achieving a predictive, well-tailored 2-D nanoarchitecture, the OTA method provides some degree of 2-D control of nanostructure.

In summary, we show a general and viable OTA approach for control of 2-D nanostructure in conducting polymers. Described
Scheme 1. (A) Lamellar Structure of the $(C T A)_{2} \mathrm{~S}_{2} \mathrm{O}_{8}$ Complex Micelle and (B) Individual (CTA $)_{2} \mathrm{~S}_{2} \mathrm{O}_{8}$ Complex with Protonated Monomer $^{a}$

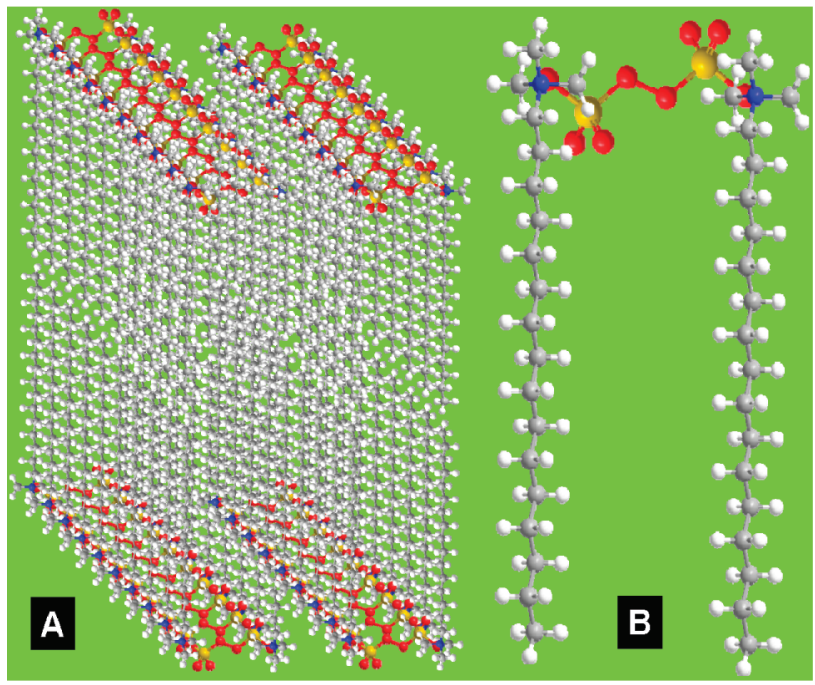

${ }^{a}$ Red, O; yellow, S; blue, N; gray, C; white, $\mathrm{H}$.

for the first time are the following: (i) the use of an oxidative template as a "universal method" to synthesize rapidly, and in one step, bulk-quantities of nanoclips of all major classes of conducting polymers, including PANI, PPy, and PEDOT; (ii) a convenient method to produce precursors that upon microwave heating yield carbon nanoclips having enhanced thermal stability after microwave heating; and (iii) a folded twin-tailed model for $(\text { CTA })_{2} \mathrm{~S}_{2} \mathrm{O}_{8}$ complex whose lamellar packing orchestrates nanoclip formation.

Acknowledgment. We gratefully acknowledge financial support from Department of Commerce, Auburn University, the University of Massachusett Lowell, and helpful discussions with Dr. Roch Chan-Yu-King.

Supporting Information Available: Synthesis, process details, and Figures S1-S6. This material is available free of charge via the Internet at http://pubs.acs.org.

\section{References}

(1) Cepak, V. M.; Martin, C. R. Chem. Mater. 1999, 11, 1363-1367. Misoska, V.; Price, W.; Ralph, S.; Wallace, G. Synth. Met. 2001, 121, 1501-1502. Simmons, M. R.; Chaloner, P. A.; Armes, S. P. Langmuir 1995, 11, 42224224. Wu, C. G.; Bein, T. Stud. Surf. Sci. Catal. 1994, 84, 2269-2276. Yu, L.; Lee, J.-I.; Shin, K.-W.; Park, C.-E.; Holze, R. J. Appl. Polym. Sci. 2003, 88, 1550-1555. Zhong, W.; Deng, J.; Yang, Y.; Yang, W. Macromol. Rapid Commun. 2005, 26, 395-400. Surwade, S. P.; Agnihotra, S. R.; Dua, V.; Manohar, N.; Jain, S.; Ammu, S.; Manohar, S. K. J. Am. Chem. Soc. 2009, 131, 12528-12529. Surwade, S. P.; Manohar, N.; Manohar, S. K. Macromolecules 2009, 42, 1792-1795.

(2) Zhang, X.; Zhang, J.; Liu, Z.; Robinson, C. Chem. Commun. 2004, $1852-$ 1853.

(3) Wu, A.; Kolla, H.; Manohar, S. K. Macromolecules 2005, 38, 7873-7875.

(4) Huang, J.; Virji, S.; Weiller, B. H.; Kaner, R. B. J. Am. Chem. Soc. 2003, $125,314-315$.

(5) Zhang, X.; Goux, W. J.; Manohar, S. K. J. Am. Chem. Soc. 2004, 126, 4502. Zhang, X.; MacDiarmid, A. G.; Manohar, S. K. Chem. Commun. 2005, 42, 5328-5330.

(6) Zhang, X.; Manohar, S. K. J. Am. Chem. Soc. 2004, 126, 12714-12715.

(7) Wang, Y.; Yu, C.; Li, Z.; Zhou, D.; Chen, W.; Xue, G. Colloid Polym. Sci. 2009, 287, 1325-1330.

(8) Wang, T.; Zhong, W.; Ning, X.; Wang, Y.; Yang, W. J. Appl. Polym. Sci. 2009, 114, 3855-3862.

(9) Ackermann, J.; Videlot, C.; Nguyen, T. N.; Wang, L.; Sarro, P. M.; Crawley, D.; Nikolic, K.; Forshaw, M. Appl. Surf. Sci. 2003, 212-213, 411-416.

(10) Zhang, X.; Zhang, J.; Song, W.; Liu, Z. J. Phys. Chem. B 2006, 110, 11581165 .

(11) Wang, Y.-G.; Li, H.-Q.; Xia, Y.-Y. Adv. Mater. 2006, 18, 2619-2623.

(12) Zhang, X.; Manohar, S. K. Chem. Commun. 2006, 2477-2479.

JA105966C 